\title{
Tendência temporal da esquizofrenia: um estudo realizado no âmbito hospitalar
}

\author{
Schizophrenia temporal trend: a study in a hospital setting
}

Aurigena Antunes de Araújo Ferreira', Gustavo Segundo de Sena², Marcus Vinicius Medeiros Galvão², Ricardo Humberto de Miranda Felix², Roberto Moreno Mendonça², Gerlane Coelho Bernardo Guerra', Francisco das Chagas Rodrigues ${ }^{3}$

\section{RESUMO}

Objetivo: Verificar as variações quanto a sexo, idade, número e tempo de internação em pacientes com esquizofrenia. Métodos: $\mathrm{O}$ estudo foi realizado no Hospital Doutor João Machado, referência estadual psiquiátrica, situado em Natal/RN - Brasil, no período de 1999 a 2005. O número de casos, o tempo de internação, a idade e o sexo foram coletados. Esses dados foram analisados pela estatística descritiva e Teste de Mann-Whitney com nível de significância de 5\%. Resultados: Durante esse tempo, 12.732 pessoas foram internadas. Os portadores de esquizofrenia corresponderam a 35,35\% (4.501). A permanência no hospital foi de 44,33 $\pm 36,9$ dias, diferente daquela observada nos não-portadores de esquizofrenia, 29,5 $\pm 61,6$ dias $(p<0,0001)$. A idade média foi de 38,7 $\pm 13,5$ anos, sendo a maioria dos pacientes do sexo masculino (65,78\%). Em 1999, de cada 100 internados, 39,8 eram portadores de esquizofrênia; em 2005 essa proporção reduziu-se para 30,1. A esquizofrenia paranóide foi a

\section{Palavras-chave}

Esquizofrenia,

epidemiologia, diagnóstico. mais freqüente, ocorrendo em 1.829 (40\%) dos pacientes durante o período. Conclusões: O estudo aponta uma tendência à redução no número de doentes internados com esquizofrenia, bem como uma diminuição no tempo de internação.

\section{Key-words}

Schizophrenia, epidemiology, diagnosis.

Recebido
12/04/2007
Aprovado
$13 / 09 / 2007$

\begin{abstract}
Objective: To find out the variation range according to gender, age, number of admissions, and stay length in hospital for schizophrenic patients. Methods: The study was performed in Doutor João Machado Hospital, Natal/RN - Brazil from 1999 through 2005. The number of cases, the stay length in hospital, the gender, and the age of the mentioned group were collected. This data was analyzed by descriptive statistics and Mann-Whitney Test with a 5\% significance level. Results: Over that time, 12,732 people were admitted. The schizophrenic patients corresponded to $35.35 \%$ $(4,501)$. The stay length in the hospital was $44.33 \pm 36,9$ days, compared to $29.5 \pm 61.6$ days for non-schizophrenic patients $(p<0.0001)$. The average age was $38.7 \pm 13.5$ years and most patients (65.78\%) were male. In 1999, 39.8 out of 100 admitted patients were schizophrenic; in 2005 this ratio was reduced to 30.1. Paranoid schizophrenia was the most prevalent type, reaching 1,829 (40\%) patients during the study time. Conclusions: The study shows a trend to reduced number of patients admitted due to schizophrenia, as well as a reduced stay length in the hospital.
\end{abstract}

1 Departamento de Biofísica e Farmacologia da Universidade Federal do Rio Grande do Norte (UFRN).

2 Faculdade de Medicina UFRN.

3 Departamento de Medicina Clínica UFRN.

Endereço para correspondência: Aurigena Antunes de Araújo Ferreira

Av. Sen. Salgado Filho s/n, Campus Universitário - Centro de Biociências - Departamento de Biofísica e Farmacologia, Lagoa Nova 59072-970 - Natal, RN

Fone: (84) 9103-2693

E-mail: aurigena@ufrnet.br 


\section{INTRODUÇÃO}

Aproximadamente 20 milhões de pessoas sofrem de esquizofrenia no mundo, o que corresponde a 1\% da população em geral; sua incidência é de 0,4 por 1.000 pessoas (Gama, 2004). Há predomínio da ocorrência em homens e mulheres entre os 10 e 50 anos de idade (Ballone, 2006).

O diagnóstico da esquizofrenia é baseado na anamnese psiquiátrica e no exame do arcabouço mental. Os sintomas característicos são classificados em duas amplas categorias: positivos e negativos. Os positivos incluem os delírios e as alucinações; os negativos, o embotamento do afeto, a anedonia e a apatia (Schiffer, 2005).

Estima-se que o componente genético represente de $70 \%$ a $80 \%$ da susceptibilidade total para desenvolver a doença. Fatores pré e perinatais ainda parecem aumentar o risco para o seu desenvolvimento. Por ser uma enfermidade complexa e comum, a esquizofrenia é, muito provavelmente, um distúrbio etiologicamente heterogêneo, isto é, devem existir casos da forma "genética" e da forma "ambiental" (Vallada Filho e Samaia, 2000).

O tratamento ao doente psiquiátrico era fundamentado em um modelo de saúde hospitalocêntrico. Manifestações contrárias surgiram nos anos 1970, constituindo o movimento de Reforma de Assistência Psiquiátrica no Brasil, contemporâneo à eclosão do "movimento sanitário". Em 1987 foram construídos os primeiros Centros Comunitários de Atenção Psicossocial (CAPS) no país, cujo propósito era a desospitalização do portador de doença mental. Em 2001, no contexto da promulgação da Lei no 10.216 e da realização da III Conferência Nacional de Saúde Mental, a política do governo federal voltada para o tema, alinhada com as diretrizes da Reforma de Assistência Psiquiátrica, passou a consolidar-se, ganhando maior sustentação e visibilidade (Brasil, 2005). Segundo dados do Conselho Nacional de Secretarias Municipais de Saúde (Bessa, 2007) funcionam hoje no país 1.016 Centros de Atenção Psicossocial, o que materializa a tendência para o tratamento dos pacientes psiquiátricos fora dos muros das instituições hospitalares.

Em virtude dos avanços alcançados pelo processo de desinstitucionalização dessas pessoas e das inovações terapêuticas ocorridas no fim da década de 1990, o estudo tem o objetivo de verificar, dentro do contexto hospitalar, as variações quanto ao número e ao tempo de internação dos portadores de esquizofrenia, a média de idade e o efeito do gênero nesta doença.

\section{MÉTODOS}

Trata-se de um estudo do tipo observacional descritivo retrospectivo, realizado no Hospital Psiquiátrico Doutor João
Machado, que está localizado no município do Natal/RN. Dentre os cinco hospitais psiquiátricos existentes no estado, este é considerado o maior e de referência. A população estudada foi formada por pacientes internados com algum tipo de esquizofrenia. O diagnóstico deste transtorno, na instituição citada anteriormente, é dado com base nos critérios da CID-10 da Organização Mundial de Saúde (OMS). Este trabalho foi submetido e aprovado pelo Comitê de Ética da Universidade Federal do Rio Grande do Norte e seu número de protocolo é 001/06 - CEP/UFRN.

\section{Variáveis}

Foram avaliadas a idade, o sexo e o tempo de internação dos pacientes. Os dados foram coletados dos arquivos dos prontuários de todos os internos com esquizofrenia, no período de 1999 a 2005.

\section{Critérios de inclusão}

Foram incluídos na pesquisa os doentes cujos prontuários de atendimento contiveram os campos para as variáveis mencionadas, preenchidos de modo legível e organizado. A estimativa de incidência do distúrbio foi obtida por intermédio de dados de primeira admissão hospitalar no período analisado.

\section{Análise dos dados}

Os dados coletados foram tabulados em planilhas, que continham os campos de preenchimento para as variáveis. Posteriormente, cada ano foi visto, tanto separadamente quanto em conjunto, para que o material de interesse fosse contabilizado e agrupado. As informações foram submetidas à estatística descritiva, ao cálculo de freqüência percentual e ao coeficiente de incidência. Para a idade e o tempo de internamento foram utilizados a média e o desvio-padrão. A diferença neste tempo para os portadores de esquizofrenia e os não-portadores foi verificada pelo teste de Mann-Whitney, com nível de significância de 5\%.

\section{RESULTADOS}

De 1999 a 2005, 12.732 pessoas foram internadas no Hospital Doutor João Machado apresentando algum transtorno psiquiátrico, e destas, 35,35\% (4.501) apresentavam esquizofrenia. A incidência deste transtorno para cada 100 doentes internados apontou a seguinte tendência: máxima em 1999 com 39,82 casos, 37,53 em 2000, 32,43 em 2001, 33,26 em 2002, 36,84 em 2003, 37,38 em 2004 e 30,11 em 2005, sendo este o menor valor encontrado.

O tempo médio de internamento foi maior em 1999, tanto para os não-portadores de esquizofrenia, 34,2 $\pm 26,7$ dias, quanto para os internados por esquizofrenia, $50 \pm 36,2$ dias. Para os primeiros a permanência mínima no hospital ocorreu em 2004, 25,1 $\pm 24,5$ dias. Já os outros atingiram uma 
permanência mínima de $37 \pm 33,5$ dias no ano de 2005. Em média, durante o período de 1999 a 2005, os não-portadores de esquizofrenia passaram 29,5 dias internados e os portadores de esquizofrenia, 44,3 dias (Figura 1). Esta diferença mostrou-se estatisticamente significantiva $(p<0,0001)$.

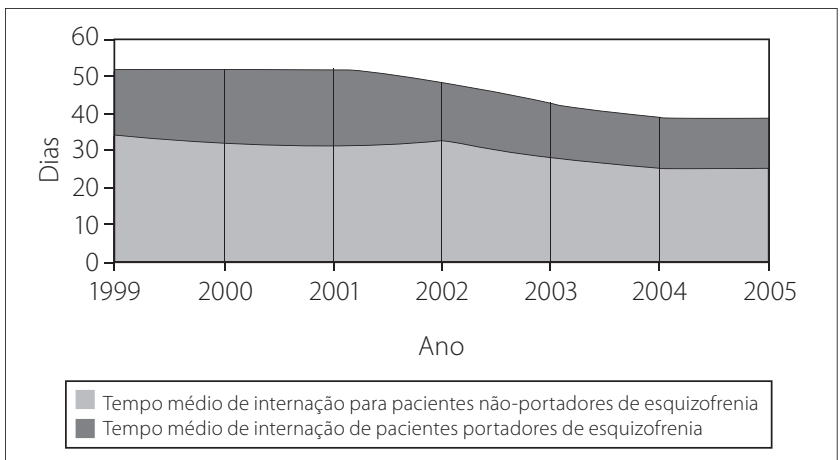

Figura 1. Tempo médio de internação de portadores e não-portadores de esquizofrenia no Hospital Doutor João Machado durante o período de 1999 a 2005. Teste de MannWhitney, $p<0,05$. Natal, RN, 2007 ( $p<0,0001)$

Em geral, a média de idade dos portadores de esquizofrenia foi de 38,7 anos. De 2000 a 2005, como aponta a figura 2, a média de idade ficou entre 37 e 40 anos, já em 1999 foi de aproximadamente 50 anos.

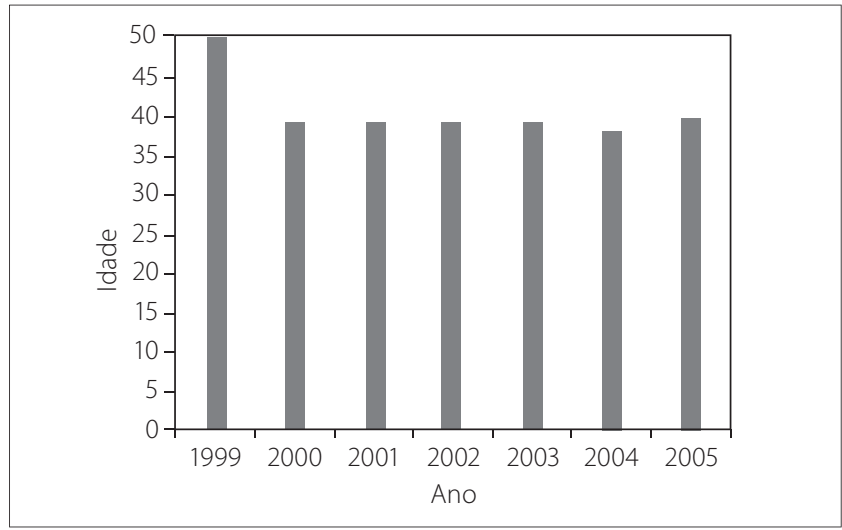

Figura 2. Média de idade de portadores de esquizofrenia internados no Hospital Doutor João Machado durante o período de 1999 a 2005. Natal, RN, 2007

Durante todo o período, a população masculina foi a que mais apresentou a psicose em questão, 65,7\% (2.961) de todos os pacientes internados. A proporção evoluiu lentamente de 64,2\%, em 1999, até atingir um máximo de 69,7\%, em 2005 (Figura 3).

Observando a distribuição das formas de esquizofrenia, o resultado demonstra que a paranóide - F20,0 (40\% - 1.829 pacientes) foi a mais freqüente durante o período de 1999 a 2005, sendo seguida pela residual - F20,5 (13\% - 568 pacientes), simples - F20,2 (11\% - 494 pacientes) e não-especificada - F20,9 (11\% - 488 pacientes). A depressão pós-esquizofrenia - F20,4 (1\% - 48 pacientes) foi o acometimento a menos freqüente (Figura 4).

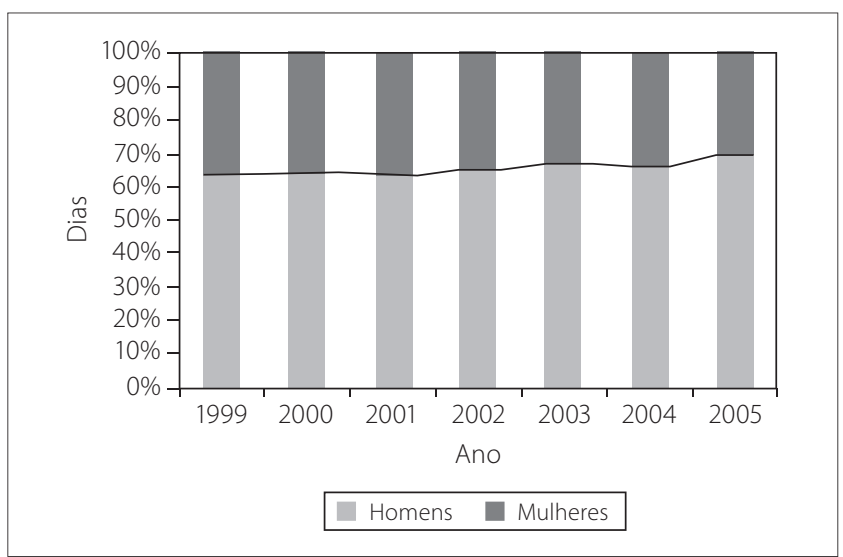

Figura 3. Contribuição dos gêneros sobre portadores de esquizofrenia internados no Hospital Dr. João Machado, durante o período de 1999 a 2005. Natal, RN, 2007

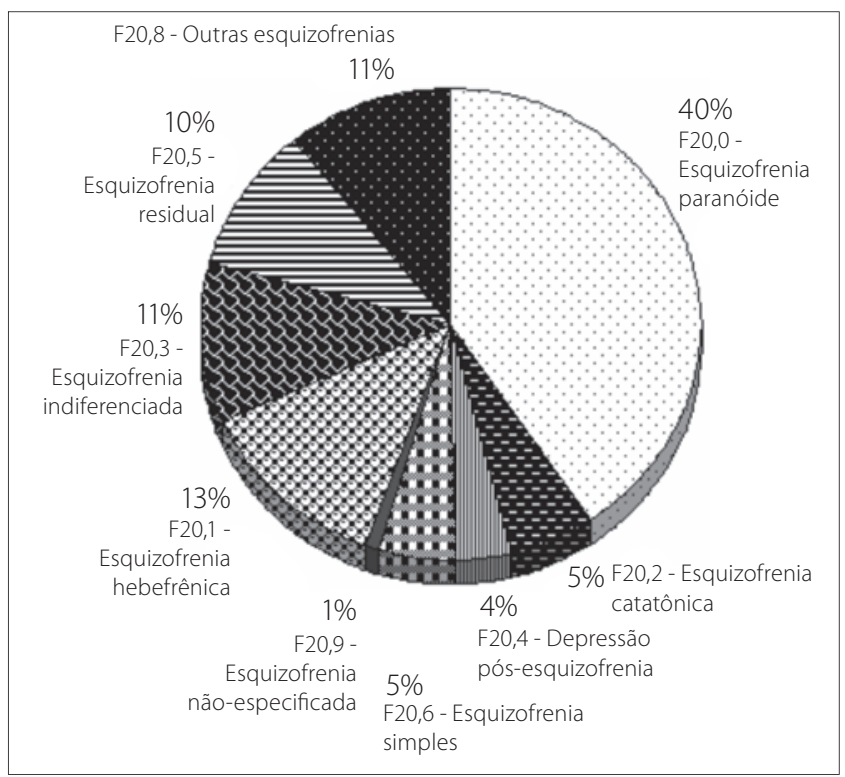

Figura 4. Distribuição das formas de esquizofrenias sobre portadores internados no Hospital Doutor João Machado, durante o período de 1999 a 2005. Natal, RN, 2007

\section{DISCUSSÃO}

De acordo com Mari e Leitão (2000), casos novos de esquizofrenia raramente ocorrem antes da puberdade e acima dos 50 anos. Corroborando, Tengan e Maia (2004) afirmam que a incidência da doença em crianças e jovens é rara. No presente estudo, a idade média dos pacientes de esquizofrenia internados obedeceu a uma tendência de permanecer em torno de 39 anos, excetuando-se o ano de 1999, no qual foi de aproximadamente de 50 anos.

Atentando para a contribuição dos sexos sobre os portadores de esquizofrenia verifica-se, como já era de se esperar, de acordo com trabalhos publicados, predomínio do sexo masculino. Walsh (1969) identificou em seu trabalho em Dublin, durante o ano de 1962, que a incidência da en- 
fermidade era de 5,7 por 10.000 habitantes nos homens e 4,6 por 10.000 nas mulheres. Caetano (1982) calculou a taxa de primeira internação em hospitais brasileiros e encontrou um maior número de homens (1,9 a 3,9 por 10.000 habitantes) em relação às mulheres (1,8 a 3,2 por 10.000).

Um estudo feito por Hamada et al. (2006), na ilha de Tsushima em Nagazaki, no Hospital Sonoda, ratifica esse fato com a incidência de 2,5 para o sexo masculino e 2,1 para o feminino por 10.000 habitantes. Isso pode ser atribuído a maior exposição dos homens ao abuso de substâncias - como álcool, tabaco e maconha - e/ou a menor adesão ao tratamento, dentre outros motivos que podem precipitar uma crise psicótica, como foi visto no estudo de Hambrecht e Häfner (1996). Pawsey e Castle (2006) afirmam que $36,3 \%$ dos homens portadores de esquizofrenia abusam do álcool, ao passo que apenas 15,7\% das mulheres o fazem; a proporção no abuso de drogas ilícitas também é maior entre o gênero masculino: 38,7\% contra 17\%. Henriques (1997) realizou um trabalho na região de Castelo Branco, em Portugal, que revelou um predomínio de homens em relação às mulheres: $211(59,3 \%)$ para $145(40,7 \%)$ casos.

Como afirma Chaves (2000), o sexo é um importante fator preditivo no curso e na evolução da esquizofrenia. Häfner (1995) considera que o gênero é um fator protetor para as mulheres por causa dos estrógenos, que atenuam a ação dopaminérgica, em virtude da redução da sensibilidade destes receptores. Os episódios agudos de esquizofrenia em pacientes do sexo feminino aumentam nas fases do ciclo menstrual, quando as concentrações basais do hormônio estão diminuídas.

Vários estudos, como os de Silveira et al. (2002) e Souza et al. (2005), apontam que a esquizofrenia paranóide foi a forma mais freqüente, corroborando os achados desta análise. Entretanto, indo ao encontro dos trabalhos referidos, o subtipo residual assumiu um papel importante, atingindo 13\% da população. Muito relevante foi também a esquizofrenia simples (11\%) e a não-especificada (11\%). Já a hebefrênica contribuiu com apenas 5\%, não assumindo um lugar de destaque nos estudos citados.

Uma freqüência aumentada da forma não-especificada - F20,9 (Figura 4) revela a ausência de critérios clínicos, no momento do atendimento, capazes de levar o médico a caracterizar especificamente a forma apresentada.

No Brasil, depois da reestruturação da atenção à saúde mental, os hospitais psiquiátricos progressivamente têm deixado de constituir a base do sistema assistencial, abrindo espaço para uma rede de serviços extra-hospitalares de crescente complexidade. Contudo, a internação psiquiátrica, a despeito das múltiplas controvérsias, continua sendo um recurso terapêutico indispensável (embora pontual) para muitos pacientes, sobretudo os mais graves (Dalgalarrondo; Botega; Banzato, 2003).
Dalgalarrondo et al. (2003) verificaram que dos 1.463 doentes internados em uma Unidade Psiquiátrica de Hospital Geral, no período 1986-1997, 23,5\% eram portadores de esquizofrenia. No estudo de Gomes et al. (2002) na Casa de Saúde Doutor Eiras/Paracambi (CSDE-P), o principal diagnóstico encontrado nos prontuários foi de esquizofrenia, com 53,6\%. No presente estudo verificou-se que este transtorno foi o diagnóstico mais freqüente entre os pacientes internados no período de 1999 a 2005, abrangendo 35,35\% dos internos.

Embora este distúrbio seja a doença psíquica de maior prevalência na população, alguns trabalhos, como o de Suvisaari et al. (1999) realizado na Finlândia, verificam um declínio na incidência de 1954 a 1965. Estes achados foram corroborados por Der et al. (1990) ao verificar redução no surgimento da esquizofrenia na Inglaterra de 1965 a 1986. Por sua vez, ao realizar uma revisão da literatura, MunkJorgensen (1995) constatou uma tendência à diminuição de novos casos nos últimos 40 a 50 anos na Europa. No entanto, verificando uma comparação entre o primeiro e o último diagnóstico observou que 35,5\% dos pacientes diagnosticados inicialmente como portadores de esquizofrenia receberam outro diagnóstico no segundo exame. Por outro lado Smith et al. (2006) verificou aumento da incidência em imigrantes da Europa, atribuindo como fatores associados o alto índice de abuso de substâncias químicas.

Pode-se verificar redução na incidência e no período de internação para os pacientes com esquizofrenia. Em 1999, os doentes internados pela psicose permaneciam $50 \pm 36,2$ dias. Em 2005, o tempo foi de $37 \pm 33,5$ dias. Observa-se que esta diminuição tende a aproximar o tempo de permanência no hospital dos portadores de esquizofrenia com a dos não-portadores, sendo essa variação estatisticamente significativa ( $p<0,0001$ ). Na pesquisa de Lima e Botega (2001), o intervalo temporal por internação foi em torno de 35,7 dias ( $D P=17,8)$. No Japão, o tempo médio para pacientes de esquizofrenia em 1996 era de 330 dias (Imai et al. 2002).

Alguns fatores podem estar relacionados com esta casuística, um deles seria a introdução dos antipsicóticos atípicos, ocorrida no Brasil ao fim da década de 1990; o acesso a eles foi regulamentado pela Portaria no 846, de 06 de novembro de 2002. Tais medicamentos alcançam aproximadamente $20 \%$ dos pacientes com refratariedade aos antipsicóticos típicos (Gama et al., 2004), bem como atendem àqueles que apresentam intolerância aos efeitos adversos destes medicamentos (típicos); dos quais 48\% abandonam o tratamento (Rosa et al., 2005), o que contribui para a elevação do número de internações hospitalares.

Outro componente macro, e possivelmente o mais significativo de todos, foi o Movimento de Reforma Sanitária iniciado na década de 1970 - que gradativamente foi se estabelecendo a partir da vigência do Sistema Único de Saúde (SUS) -, resultado de um processo de reestruturação política pelos quais passam os serviços de saúde mental no 
Brasil. O Ministério da Saúde cria linhas específicas de financiamento para os serviços abertos substitutivos ao hospital psiquiátrico. Novos mecanismos são desenvolvidos para a fiscalização, a gestão e a redução programada de leitos psiquiátricos no país (Brasil, 2005).

A interação entre governo, sociedade e serviços de saúde constitui um tripé que almeja novas dimensões e expectativas quanto à qualidade de vida do paciente psiquiátrico. Este, que antes era marginalizado e excluído da sociedade, pelo seu confinamento nos grandes manicômios, atravessa gradativamente os muros das instituições hospitalares e insere-se em um contexto social de modo que reduz as diferenças, estigmas e desigualdades.

\section{CONCLUSÕES}

O presente estudo constatou que a média de idade dos pacientes internados era de 39 anos, a maior freqüência de internações era do sexo masculino, e o maior número de pacientes apresentava esquizofrenia paranóide. Pode-se verificar redução no número e no tempo de internação, o que aponta para a importância de o Estado brasileiro assistir o paciente integralmente.

\section{AGRADECIMENTOS}

Agradecemos a Rodrigo Bandeira, Rodrigo Tibúrcio, Carlo Victor Azevedo e Ângelo José Pimentel, acadêmicos de medicina da Universidade Federal do Rio Grande do Norte (UFRN), pela colaboração física e intelectual mais que necessária. Também a Eduardo Cruz, Lígia Leite e Cláudio Tamires pelas suas contribuições com a língua inglesa. E ao apoio financeiro, de suma importância para a realização deste projeto: Departamento de Ciência e Tecnologia (DECIT), Secretaria de Ciência, Tecnologia e Insumos Estratégicos (SCTIE), Ministério da Saúde (MS), Ministério de Ciências e Tecnologia (MCT) e Conselho Nacional de Desenvolvimento Científico e Tecnológico (CNPq)

\section{REFERÊNCIAS}

Ballone GJ. Esquizofrenias [acesso em 2006 Jul 29]. Disponível em: http://virtualpsy.locaweb. com.br/index.php?art=171\&sec $=54$.
Bessa S. 0 não-lugar da loucura. Rev Conasems, 22:19-24, 2007.

Brasil. Ministério da Saúde. Secretaria de Atenção à Saúde. DAPE. Coordenação Geral de Saúde Mental. Reforma psiquiátrica e política da saúde mental no Brasil. Documento apresentado à Conferência Regional de Reforma dos Serviços de Saúde Mental: 15 anos depois de Caracas. OPAS. Brasília, 2005.

Caetano R. Adimisiones de primer ingreso a los servicios psiquiátricos en Brasil, 1960-1974. Boletín de la Oficina Sanitaria Panamericana 92: 103-117, 1982.

Chaves AC. Diferenças entre os sexos na esquizofrenia. Rev Bras Psiquiatr, 22:21-22, 2000.

Dalgalarrondo P, Botega NJ, Banzato CEM. Pacientes que se beneficiam de internação psiquiátrica em hospital geral. Rev Saúde Pública, 37:629-634, 2003.

Der G, Gupta S, Murray RM. Is schizophrenia disappearing? Lancet, 335:513-516, 1990.

Gama CS, Souza CM, Lobato Ml et al. Relato do uso de clozapina em 56 pacientes atendidos pelo Programa de Atenção à Esquizofrenia Refratária da Secretaria da Saúde e do Meio Ambiente do Estado do Rio Grande do Sul. Rev Psiquiatr do Rio Grande do Sul, 26:21-28, 2004.

Gomes MPC et al. Censo dos pacientes internados em uma instituição asilar no Estado do Rio de Janeiro: dados preliminares. Cad Saúde Pública, 18:1803-1807, 2002.

Häfner H. Epidemiology of schizophrenia. The disease model of schizophrenia in the light of current epidemiological knowledge. Euro Psychiat, 10:217-227, 1995.

Hamada Y, Otha Y, Nakani Y. Epidemiological study of schizophrenia on the Island of Tsushima in Nagasaki Prefecture. Seishin Shinkeigaku Zasshi, 108:117-131, 2006.

Hambrecht M, Häfner H. Substance abuse and the onset of schizophrenia. Biol Psychiatry, 40:1155-1163, 1996.

Henriques F. Prevalência e Incidência das psicoses na região de Castelo Branco. Rev Saúde Amato Lusitano, 4:29-34, 1997.

Imai H, Hosoml J, Nakao H et al. Characteristics of psychiatric hospitals associated with length of stay in Japan. Health Policy, 74:115-121, 2002.

Lima MCP, Botega NJ. Hospital dia: Para quem e para quê? Rev Bras Psiquiatr, 23:195-199, 2001.

Mari JJ, Leitao RJ. A epidemiologia da esquizofrenia. Rev Bras Psiquiatr, 22:15-17, 2000.

Munk-Jorgensen P. Decreasing rates of incident schizophrenia cases in psychiatric service: a review of the literature. Euro Psychiatry, 10:129-141, 1995.

Pawsey B, Castle D. Substance use and psychosis. Aust Fam Physician, 35(3):110-2, 2006.

Rosa MA, Marcolin MA, Elkis H. Avaliação dos fatores de aderência ao tratamento medicamentoso entre pacientes brasileiros com esquizofrenia. Rev Bras Psiquiatr, 27:178-184, 2005.

Schiffer RB. Os distúrbios psiquiátricos na prática médica. In: Goldman L, Ausiello D. Cecil: Tratado de medicina interna. Elsevier, p. 2587-2599, São Paulo, 2005.

Silveira C, Rolnik D, Monteiro T et al. Perfil clínico e sociodemográfico de pacientes com esquizofrenia de início recente admitidos em serviço hospitalar em São Paulo. Rev Bras Psiquiatr, 24:63-78, 2002.

Smith GN, Boydell J, Murray RM et al. The incidence of schizophrenia in European immigrants to Canada. Schizophr Res, 87:205-211, 2006.

Souza JC, Souza N, Caetano D. Tempo médio de hospitalização em um hospital psiquiátrico de Campo Grande, Mato Grosso do Sul. J Bras Psiquiatr, 54:13-18, 2005.

Suvisaari JM, Haukka JK, Tanskanen AJ et al. Decline in the incidence of schizophrenia in Finnish cohorts born from 1954 a 1965. Arc Gen Psychiatry, 56:733-740, 1999.

Tengan SK, Maia AK. Psicoses funcionais na infância e adolescência. J Pedriatr, 80:03-10, 2004.

Vallada Filho HP, Samaia H. Esquizofrenia: aspectos genéticos e estudos de fatores de risco. Rev Bras Psiquiatr, 22:02-04, 2000.

Walsh D. Mental illness in Dublin: first admissions. Br J Psychiatry, 115:449-56, 1969. 